\title{
Seminare 2005
}

\section{Röntgen in der Arztpraxis}

\section{Teilnehmende}

Das Seminar richtet sich an Ärztinnen und Ärzte mit bestehender Praxis und an solche, die vor einer Praxiseröffnung oder Praxisübernahme stehen.

\section{Themen}

Das Seminar vermittelt einerseits Kenntnisse über die gesamte bestehende Röntgenthematik, andererseits werden klare Trends für die Praxis der nächsten Generation mit digitalem Röntgen aufgezeigt. Als spezifische Themen werden behandelt: Röntgentarife nach TARMED, Rentabilität Röntgen in der Arztpraxis, bestehende und neue Vorschriften ab 1. Januar 2008 (kann mit einem Wechsel der Anlage verbunden sein; Achtung bei Praxisübernahmen). Zudem wird einerseits eine Checkliste vorgestellt und durchgearbeitet für die Evaluation und die Beschaffung einer neuen Anlage, andererseits werden Bewertungskriterien für die Übernahme einer Röntgenanlage (bei Praxisübernahmen) aufgezeigt. Die Seminarteilnehmer erstellen und bearbeiten innerhalb des Seminars digitale Röntgenbilder.

\section{Unterlagen}

Die Seminarteilnehmer/innen erhalten ein Skript mit Zahlen einer Arztpraxis mit Kostenberechnung, Amortisation und interessanten Leasingformen (ab welcher Auslastung arbeite ich kostendeckend oder mit Gewinn?). Zudem erhalten sie eine Checkliste für die Evaluation und die Beschaffung einer neuen Röntgenanlage sowie eine CD-ROM oder ein Fachbuch mit über 50 Modellaufnahmen.

\section{Kosten}

Fr. 300.- (inkl. sämtlicher Kursunterlagen und Verpflegung).

\section{Daten}

\begin{tabular}{|c|c|c|c|}
\hline \multirow[t]{2}{*}{ Nr. 17} & Donnerstag, 1 & 19. Mai 2005 & \multirow{2}{*}{$\begin{array}{l}\text { Ausbildungs- } \\
\text { zentrum } \\
\text { Galexis }\end{array}$} \\
\hline & Cham & 9.30-16.00 Uhr & \\
\hline Nr. 18 & $\begin{array}{l}\text { Donnerstag, } \\
\text { Burgdorf }\end{array}$ & $\begin{array}{l}\text { 1. September } 2005 \\
9.30-16.00 \mathrm{Uhr}\end{array}$ & $\begin{array}{l}\text { Typon } \\
\text { Imaging AG }\end{array}$ \\
\hline
\end{tabular}

\section{Anmeldung}

Das Seminar ist auf maximal 15 Personen beschränkt. Sie können sich bei nachfolgend aufgeführter Adresse (FMH Consulting Services) oder via Internet unter www.fmhservices.ch einschreiben.

\section{Das Labor in der Arztpraxis}

\section{Teilnehmende}

Das Seminar richtet sich an Praxiseröffner/innen, Praxisübernehmer/innen sowie an bereits praxistätige Ärztinnen und Ärzte.

\section{Themen}

Gesetzliche Überlegungen für das Betreiben eines Praxislabors (FAPL, interne und externe Qualitätskontrolle); betriebswirtschaftliche Gesichtspunkte (Geräteanschaffung, laufende Unkosten, Platzbedarf, Personalbindung, Ausund Weiterbildung vs. Vergütung gemäss ALT); fachliche Gesichtspunkte (Ergänzung Praxislabor/Fachlabor, Präsenzdiagnostik, Qualität der Analysen); welche Dienstleistungen können vom externen Labor erwartet werden (mögliche Serviceleistungen, Befundübermittlung, Kostenstatistik)?; Demonstration der wichtigsten Praxislaborgeräte in der Schweiz.

\section{Unterlagen}

Die Seminarteilnehmer/innen erhalten einen Seminarordner mit Unterlagen zu den behandelten Themen.

\section{Kosten}

Das Seminar ist für FMH-Mitglieder gratis. Die Kosten werden von der Labornetz daCapo gedeckt.

\section{Daten}

$\begin{array}{llll}\text { Nr. } 20 & \text { Donnerstag, 14. April 2005 } & \text { FMT } \\ & \text { Zürich } & 9.30-16.30 \mathrm{Uhr} & \text { Zürich }\end{array}$




\section{Patientengerechtes Verhalten in der Arztpraxis}

Teilnehmende

Das Seminar richtet sich an das Medizinische Praxispersonal sowie an Ärztinnen und Ärzte.

\section{Inhalt}

Der erste Eindruck einer Patientin bzw. eines Patienten, welchen sie bzw. er von der Praxis vermittelt bekommt, ist für den Praxiserfolg sehr wichtig. Den Seminarteilnehmern/-innen wird aufgezeigt, wie dem Patienten ein «Kundengefühl» vermittelt wird und wie Reklamationen erledigt werden. Es wird darauf eingegangen, welches die Möglichkeiten des Telefons, als akustische Visitenkarte, und des persönlichen Patientenkontakts sind.

\section{Kosten}

- Für Mitglieder des Schweizerischen Verbandes Medizinischer Praxis- Assistentinnen: Fr. 285.-

- Für übrige Teilnehmer/innen (MPA ohne Verbandsmitgliedschaft, Ärztin/Arzt): Fr. 300.-

\section{Daten}

\begin{tabular}{llrl} 
Nr. 21 & $\begin{array}{l}\text { Donnerstag, 12. Mai } 2005 \\
\text { Zürich }\end{array}$ & FMT Zürich \\
\hline Nr. 22 & $\begin{array}{l}\text { Donnerstag, 27. Oktober 2005 } \\
\text { Bern }\end{array}$ & 9.00-17.45 Uhr & Hotel Bern \\
& &
\end{tabular}

\section{Anmeldung}

Sie können sich bei nachfolgend aufgeführter Adresse (FMH Consulting Services) oder via Internet unter www.fmhservices.ch einschreiben.

\section{Biostrukturanalyse für mehr Erfolg in der Arztpraxis}

\section{Teilnehmende}

Das Seminar richtet sich an sämtliche Ärztinnen und Ärzte sowie an medizinisches Praxispersonal.

\section{Inhalt}

In unserer hochtechnisierten Medizinwelt haben wir leider vergessen, dass der EQ (emotionale Quotient) im Umgang mit unserer Familie, unseren Freunden, unserem Team und unseren Patienten eine viel wichtigere Rolle spielt als der IQ. Das gute Zusammenspiel Ihres Teams sowie das Vertrauen Ihrer Patienten hängt von Ihrem EQ ab!
In diesem eintägigen Seminar geben Sie keine dunklen Geheimnisse von sich preis, sondern Sie analysieren Ihre Stärken und Schwächen im täglichen Umgang mit Ihrem Team und Ihren Patienten. Sie erhöhen Ihre Sozialkompetenz und lernen Strategien für eine noch bessere $\mathrm{Zu}$ sammenarbeit und wie Sie noch gezielter auf die Bedürfnisse Ihrer Patienten eingehen können. Das gegenseitige Verständnis wächst und Sie verstärken dadurch die Kunden- bzw. Patientenbindung.

Zusätzliche Infos unter www.structogram.info.

\section{Unterlagen}

Die Seminarteilnehmenden erhalten die Bücher «Die Biostruktur-Analyse 1: Schlüssel zur Selbstkenntnis» und «Die Biostruktur-Analyse 2: Schlüssel zur Menschenkenntnis» von Rolf W. Schirm sowie Arbeitsblätter.

\section{Kosten}

Fr. 550.- (inkl. sämtlicher Kursunterlagen und Verpflegung).

\section{Daten}

$\begin{array}{llrl}\text { Nr. 23 } & \begin{array}{l}\text { Donnerstag, 21. April 2005 } \\ \text { Zürich }\end{array} & \text { FMT Zürich } \\ \text { Nr. } 24 & \begin{array}{l}\text { Donnerstag, 20. Oktober 2005 } \\ \text { Bern }\end{array} & \begin{array}{l}\text { Inselspital } \\ \text { 9.00-16.30 Uhr }\end{array} & \text { Bern }\end{array}$

\section{Anmeldung}

Sie können sich bei nachfolgend aufgeführter Adresse (FMH Consulting Services) oder via Internet unter www.fmhservices.ch einschreiben.

\section{Anmeldung und Auskunft}

FMH Consulting Services, Simone Köpfli, Burghöhe 1, 6208 Oberkirch, Tel. 04192500 77, Fax 0419210586 oder via Internet unter www.fmhservices.ch.

\section{Hinweis}

Bei sämtlichen Seminaren, bei welchen die Kosten teilweise oder gänzlich von Seminarsponsoren gedeckt werden, werden die Teilnehmeradressen den jeweiligen Sponsoren zur Verfügung gestellt.

\section{Annullierungsbedingungen}

Bei Abmeldungen oder Fernbleiben werden die folgenden Unkostenbeiträge erhoben:

- $30 \%$ der Seminarkosten ab 14 Tage vor Seminarbeginn;

- $\quad 100 \%$ der Seminarkosten ab 7 Tage vor Seminarbeginn oder Fernbleiben. 